\title{
Improvement of the emission current from a cesiated metal-oxide-semiconductor cathode
}

\author{
H. Mimura ${ }^{a)}$ and Y. Neo \\ Research Institute of Electronics, Shizuoka University, 3-5-1 Johoku, Hamamatsu 432-8011, Japan \\ H. Shimawaki \\ Hachinohe Institute of Technology, Hachinohe 031-8501, Japan \\ Y. Abe, K. Tahara, and K. Yokoo \\ Research Institute of Electrical Communication, Tohoku University, Katahira, Sendai 980-8577, Japan
}

(Received 6 October 2005; accepted 6 February 2006; published online 24 March 2006)

We have reduced the work function of the gate electrode of a metal-oxide-semiconductor tunneling
cathode by cesiation. After cesiation, there was a considerable increase in the emission current and
a large number of electrons were detected at energies lower than the original vacuum level of the
poly-Si gate electrode. These results indicate that almost all the tunneling electrons have energies
lower than the original vacuum level of the gate electrode, and that after cesiation electrons initially
prevented from being emitted by the original vacuum level were emitted, because of the reduced
work function due to cesiation. (c) 2006 American Institute of Physics. [DOI: 10.1063/1.2189112]

Many types of cold cathode such as Spindt-type metal emitters, ${ }^{1} \mathrm{Si}$ emitters, ${ }^{2}$ carbon nanotube emitters, ${ }^{3}$ metalinsulator-metal (MIM) emitters, ${ }^{4}$ porous $\mathrm{Si}$ emitters ${ }^{5}$ etc., have been developed for applications in vacuum nanoelectronic devices, including high-frequency ${ }^{6}$ and environment hard electronic devices, point $\mathrm{x}$-ray sources, ${ }^{7}$ and flat panel displays. ${ }^{8}$ For these applications, the cathode is required to have high current density at low extraction voltage and to be stable in operation. However, the cathode that completely satisfies these requirements has yet to be developed. A metaloxide-semiconductor (MOS) cathode is a promising candidate as a fine electron source, because it operates at a gate voltage of less than $10 \mathrm{~V},{ }^{9}$ has a fluctuation-free emission current, ${ }^{10}$ produces uniform emission from the whole emitter area, and has a highly directional electron beam. Furthermore, the cathode is almost independent of the ambient gas pressure. ${ }^{10}$ The emission mechanism of the MOS tunneling cathode is derived from the emission characteristics and energy distribution. ${ }^{9-12}$ As shown in Fig. 1, when a gate voltage exceeding the work function of the gate electrode is applied, electrons travel in the conduction band of the thin oxide layer and the gate electrode after tunneling through the potential barrier in the oxide, and some of them are emitted into the vacuum. Electrons tunneling into the conduction band of the oxide layer are hot ones. However, since they are strongly scattered in both the oxide layer and the gate electrode, their energy is widely distributed and the energy of almost all of them is reduced to less than that of the vacuum level (work function) of the gate electrode. Consequently, almost all the electrons are absorbed in the gate electrode as diode current. Hence, the only disadvantage of the MOS tunneling cathode is the low emission efficiency, i.e., the ratio of emission to diode current is less than $1 \%$. The emission mechanism suggests that there is a large number of electrons with energies below the vacuum level of the gate electrode that are not emitted into the vacuum. This means that the

\footnotetext{
${ }^{\text {a) }}$ Author to whom correspondence should be addressed; electronic mail:
} mimura@rie.shizuoka.ac.jp emission efficiency could be drastically improved by reducing the work function of the gate electrode.

In this letter, in order to improve the emission efficiency and confirm the emission mechanism we have reduced the work function of the gate electrode of a MOS tunneling cathode by cesiation. After cesiation, the emission current was appreciably increased and a considerable number of electrons with energies lower than the original vacuum level of the gate electrode were detected.

A schematic of a MOS cathode is shown in Fig. 2. An $n$-type $\mathrm{Si}(100)$ wafer with a resistivity of $4-6 \Omega \mathrm{cm}$ was used for the substrate. We used a high quality $\mathrm{SiO}_{2}$ layer as a gate oxide, ${ }^{12}$ because the reliability of the MOS cathode depends on the oxide quality. No degradation was observed during the experiments. The thicknesses of the oxide layer and the $n^{+}$-poly-Si gate electrode were 9.8 and $20 \mathrm{~nm}$, respectively. The optimum thickness of the poly-Si gate electrode was $20 \mathrm{~nm}$, because the thinner thickness decreased the emission efficiency due to lower lateral conductivity and the thicker thickness also decreased the emission efficiency due to stronger scattering. The electron emission area was 500 $\times 500 \mu \mathrm{m}^{2}$. The detailed process is described in Ref. 12. The Cs source used in the experiment was a metal wire consisting of $\mathrm{Cs}_{2} \mathrm{Cr}_{2} \mathrm{O}$ and $\mathrm{Al}(16 \%)+\mathrm{Zr}(84 \%), \mathrm{Cs}_{2} \mathrm{Cr}_{2} \mathrm{O}: \mathrm{Al}+\mathrm{Zr}=1: 5$. $\mathrm{Cs}$ was evaporated over the entire gate electrode area by running a current through the wire up to $1.8 \mathrm{~A}$ and maintain-

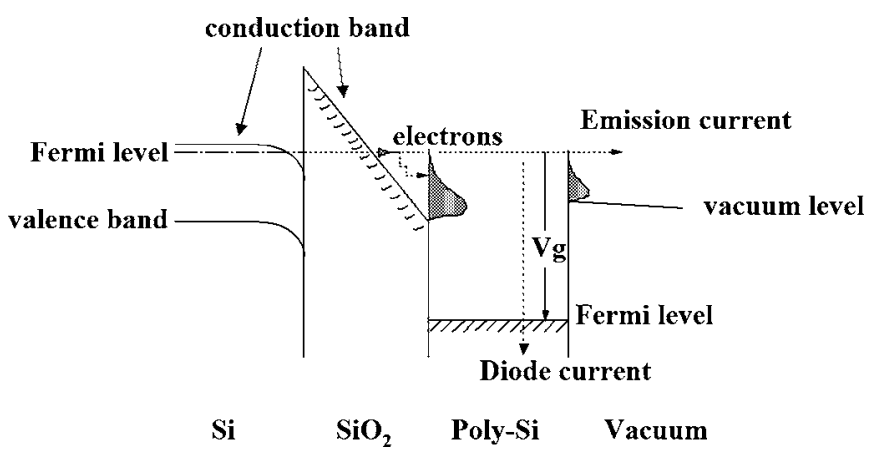

FIG. 1. Band diagram of the MOS tunneling cathode. 


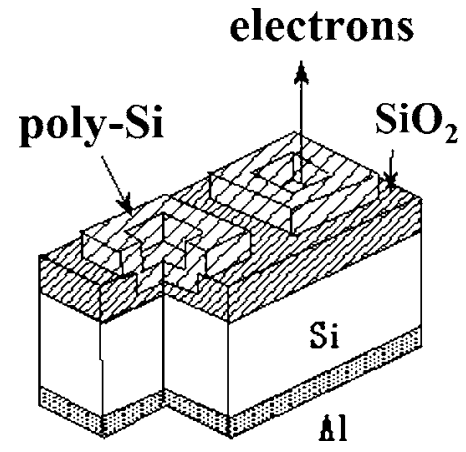

FIG. 2. Schematic of the MOS cathode.

ing this for a few seconds. During evaporation, the pressure was kept to below $1 \times 10^{-5} \mathrm{~Pa}$. In this experiment, we made no estimate of the number of atomic layers deposited. In the emission measurements, an extraction voltage was applied to the poly-Si gate electrode of the emitter, and the diode current $\left(I_{d}\right)$ and the corresponding anode current $\left(I_{e}\right)$, equivalent to the emission current, were measured as a function of the extraction voltage. The anode was an $\mathrm{Al}$ plate located $5 \mathrm{~mm}$ away from the cathode, and the voltage applied to the anode was $200 \mathrm{~V}$. To measure the energy distribution of the emitted electrons, the anode was replaced by a parallel-plate electron energy analyzer.

Figure 3 shows the diode and emission currents for the MOS cathode before and after cesiation. After cesiation, there is a significant increase in emission current. Figure 4(a) shows the energy distributions of the MOS cathode at a gate voltage of $9.4 \mathrm{~V}$ before and after cesiation, and Fig. 4(b) shows the relationship between the energy band diagram and the energy distributions. The zero point in the energy distribution corresponds to the original vacuum level of the poly-Si gate electrode. The inset in Fig. 4(a) shows the expanded energy distributions near the high-energy tail and although these coincide with each other, considerably more electrons are detected at energies lower than the original vacuum level of the poly-Si gate electrode after cesiation. This means that almost all of the tunneling electrons have energies lower than the original vacuum level of the poly-Si gate electrode as predicted by the emission mechanism of the MOS cathode, and that electrons initially prevented from emission by the vacuum level can be emitted, because of the reduction in the work function due to cesiation.

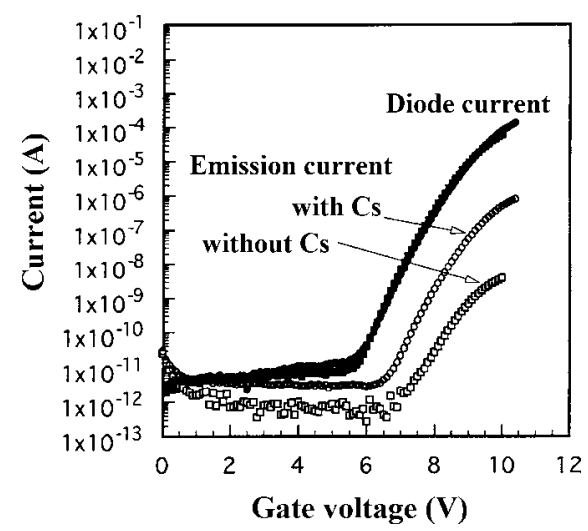

FIG. 3. Diode and emission currents for the MOS cathode before and after cesiation.

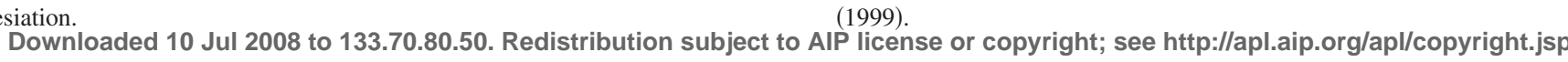

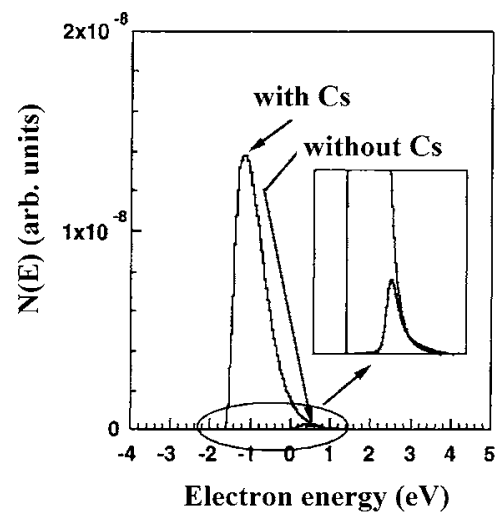

(a)

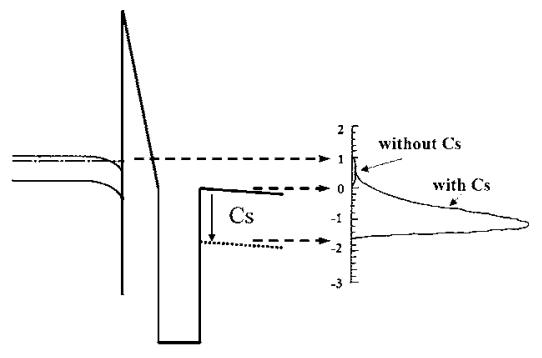

(b)

FIG. 4. (a) Energy distributions of the MOS cathode at a gate voltage of $9.4 \mathrm{~V}$ before and after cesiation, and (b) the relationship between the energy band diagram and the energy distributions.

In summary, we fabricated MOS tunneling cathodes, and measured their emission characteristics and energy distributions before and after cesiation, which was done in order to improve the emission efficiency and confirm the emission mechanism. After cesiation of the poly-Si gate electrode, an appreciable increase in the emission current was measured and a considerable number of electrons with energies lower than the original work function of the gate electrode were detected. Although the tunneling electrons in the conduction band of the oxide layer are hot ones, they are widely distributed in energy due to strong scattering in both the oxide layer and the gate electrode. Therefore, almost all tunneling electrons have energies lower than the original vacuum level of the poly-Si gate electrode. After cesiation, the electrons initially prevented from emission by the original vacuum level can be emitted into the vacuum, because the work function is reduced by cesiation. Lowering the work function of the gate electrode is the most practical method for improving the emission current.

This work was in part supported by a Grant-in-Aid for Scientific Research from the Ministry of Education, Culture, Sports, Science, and Technology, Japan.

${ }^{1}$ P. R. Schwoebel, C. A. Spindt, and C. E. Holland, J. Vac. Sci. Technol. B 23, 619 (2005).

${ }^{2}$ H. Shimawaki, K. Tajima, H. Mimura, and K. Yokoo, IEEE Trans. Electron Devices 49, 1665 (2002).

${ }^{3}$ W. Zu, C. Bower, O. Zhou, G. Kochanski, and S. Jin, Appl. Phys. Lett. 75, 873 (1999).

${ }^{4}$ T. Kusunoki and M. Suzuki, IEEE Trans. Electron Devices 47, 1667 (2000).

${ }^{5}$ T. Komoda, X. Sheng, and N. Koshida, J. Vac. Sci. Technol. B 17, 1076 
${ }^{6}$ H. Hasegawa, H. Mimura, and K. Yokoo, Jpn. J. Appl. Phys., Part 142 , 4051 (2003).

${ }^{7}$ T. Matsumoto and H. Mimura, Appl. Phys. Lett. 84, 1804 (2004).

${ }^{8}$ S. Ito, M. Tanaka, and T. Tonegawa, J. Vac. Sci. Technol. B 22, 1362 (2004).

${ }^{9}$ K. Yokoo, H. Tanaka, S. Sato, J. Murota, and S. Ono, J. Vac. Sci. Technol. B 11, 429 (1993).
${ }^{10}$ K. Yokoo, S. Sato, G. Koshita, I. Amano, J. Murota, and S. Ono, J. Vac. Sci. Technol. B 12, 801 (1994).

${ }^{11}$ K. Yokoo, G. Koshita, S. Hanzawa, Y. Abe, and Y. Neo, J. Vac. Sci. Technol. B 14, 2096 (1996).

${ }^{12}$ H. Mimura, Y. Abe, J. Ikeda, K. Tahara, Y. Neo, H. Shimawaki, and K. Yokoo, J. Vac. Sci. Technol. B 16, 803 (1998). 